\title{
Controle genético para a tolerância a geada em progênies de Eucalyptus urophylla
}

\section{Genetic control for frost tolerance in Eucalyptus urophylla progenies}

\author{
Cristiano Bueno de Moraes ${ }^{1}$, Evandro Vagner Tambarussi ${ }^{2}$, Lorena Gama ${ }^{3}$, \\ Fernanda Maria Abilio ${ }^{4}$, André Carignato ${ }^{5}$, Leo Zimback ${ }^{6}$, \\ Rinaldo César de Paula ${ }^{7}$ e Edson Mori ${ }^{8}$
}

\begin{abstract}
Resumo
A pesquisa teve como objetivo estudar o controle genético de caracteres silviculturais em progênies de Eucalyptus urophylla para a tolerância à geada. Foram instalados três testes de progênies de polinização aberta em blocos casualizados, 150 progênies, quatro repetições, seis plantas/parcela. Os testes foram implantados em duas regiões contrastantes para a ocorrência de geadas. Em áreas de geadas os testes foram implantados nas áreas das empresas Eucatex (Itatinga/SP) e Palmasola (Palma Sola/SC), e na Estação Experimental de Anhembi/SP, onde a ocorrência de geadas é rara. Altura de plantas, diâmetro à altura do peito e volume de madeira, aos 12 e 24 meses de idade foram mensuradas. A variabilidade genética na população foi alta, o coeficiente de variação genético individual variou de 5,85\% (ALT) até 22,58\% (VOL) e a herdabilidade no sentido restrito de 0,13 (ALT) até 0,77 (DAP e VOL), indicando um elevado potencial para seleção e melhoramento da espécie para a tolerância ao frio.
\end{abstract}

Palavras-chave: parâmetros genéticos; melhoramento florestal; geada.

\begin{abstract}
This research aimed to study the genetic control of silvicultural traits in Eucalyptus urophylla progenies for frost tolerance. Three open-pollinated progeny tests were installed in a randomized blocks design, 150 progenies, four replications, six plants per plot. The tests were implemented in two contrasting regions for the occurrence of frost. In areas with frost occurrence the tests were implemented in the Eucatex (Itatinga/ $\mathrm{SP}$ ) and Palmasola (Palma Sola/SC) companies; for areas with rare frosts the trial was established in Experimental Station of Anhembi/SP. Plant height; diameter at breast height and wood volume at 12 and 24 months old were measured. The genetic variability in the population was high, the individual genetic variation coefficient ranging from $5.85 \%$ (ALT) to $22.58 \%$ (VOL) and the heritability in the narrow sense from 0.13 (ALT) to 0.77 (DAP and VOL), indicating a high potential for selection and breeding of the species for frost tolerance.
\end{abstract}

Keywords: genetic parameters; progeny trial; forest tree improvement; frost.

${ }^{1}$ Professor Adjunto do Departamento de Engenharia Florestal. UFT - Universidade Federal de Tocantins, Campus de Gurupi. - 77402970 - Gurupi, TO, Brasil. E-mail: cbmoraes@uft.edu.br.

2Professor Adjunto do Departamento de Engenharia Florestal. UNICENTRO - Universidade Estadual do Centro-Oeste / Setor de Ciências Agrárias e Ambientais. PR 153 Km 7 - 84500000 - Irati, PR, Brasil. E-mail: tambarussi@gmail.com.

${ }^{3}$ Graduanda em Engenharia Florestal. UFT - Universidade Federal de Tocantins / Campus de Gurupi. 77402970 - Gurupi, TO, Brasil. E-mail: lorenagamaeng@gmail.com.

${ }^{4}$ Mestranda em Ciência Florestal. UNESP - Universidade Estadual Paulista "Júlio de Mesquita Filho" / FCA - Faculdade de Ciências Agronômicas - Campus de Botucatu. Fazenda Experimental Lageado. Rua José Barbosa de Barros, 1780 - 18610 307 - Botucatu - SP, Brasil. E-mail: fernandama@eucatex.com.br.

${ }^{5}$ Engenheiro Florestal. UNESP - Universidade Estadual Paulista "Júlio de Mesquita Filho" / FCA - Faculdade de Ciências Agronômicas - Campus de Botucatu. Fazenda Experimental Lageado. Rua José Barbosa de Barros, 1780 - 18610 -307 - Botucatu - SP, Brasil. E-mail: andrecarignatoflorestal@hotmail.com.

${ }^{6}$ Pesquisador Científico. IF - Instituto Florestal do Estado de São Paulo - Floresta Estadual de Botucatu. Avenida José Ítalo Bacchi s/n - Aeroporto - 18603-970 - Botucatu, SP, Brasil E-mail: lzimback@terra.com.br.

${ }^{7}$ Professor Adjunto do Departamento de Produção Vegetal. UNESP - Universidade Estadual Paulista "Júlio de Mesquita Filho" / FCA - Faculdade de Ciências Agrárias e Veterinárias de Jaboticabal. Via de Acesso Prof. Paulo Donato Castellane,

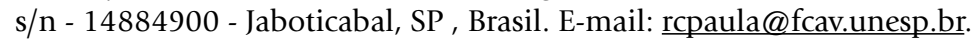

${ }^{8}$ Professor Titular no Departamento de Produção e Melhoramento Vegetal. UNESP - Universidade Estadual Paulista “Júlio de Mesquita Filho" / FCA - Faculdade de Ciências Agronômicas - Campus de Botucatu. Fazenda Experimental Lageado. Rua José Barbosa de Barros, 1780 - 18610-307 - Botucatu - SP, Brasil. E-mail: esmori@fca.unesp.br.

Sci. For., Piracicaba, v. 44, n. 110, p. 373-381, jun. 2016 DOI: dx.doi.org/10.18671/scifor.v44n110.10 


\section{INTRODUÇÃO}

Espécies do gênero Eucalyptus são plantadas em mais de 100 países tropicais e subtropicais e cumprem papel essencial para a produção sustentável de matéria prima para papel, celulose, energia e madeira sólida, dentre outros, contribuindo de forma decisiva para a preservação das florestas nativas. O Brasil é um dos principais produtores de madeira de Eucalyptus do mundo, com média de produtividade entre 45 a $50 \mathrm{~m}^{3}$.ha' ${ }^{-1}$.ano ${ }^{-1}$, sendo que em alguns casos atingem $60 \mathrm{~m}^{3}$. ha ${ }^{-1}$. ano ${ }^{-1}$ (SNIF, 2015), principalmente devido a utilização de genótipos adaptados às diferentes condições de clima existentes no país (ALMEIDA et al., 2007).

Frio, seca e alta salinidade são os estresses ambientais mais comuns que afetam a produtividade nas plantas (KAYAL et al., 2006). Dentre esses estresses, o frio está entre os fatores que limitam a produção de madeira e prejudicam a formação de plantios homogêneos de Eucalyptus na região sul do Brasil, e em outras regiões do mundo (SILVA et al., 2009; MORAES et al., 2014). Este fenômeno danifica as folhas das plantas pelo congelamento o que acarreta a inibição da fotossíntese, translocação mais lenta de carboidratos, baixas taxas respiratórias, inibição da síntese de proteínas e aumento da degradação das proteínas existentes (TAIZ; ZEIGER, 2013). Nesta situação, os danos às plantas vão desde queda das folhas, morte do ponteiro apical ou até morte dos indivíduos pelo frio intenso (TIBBITS; HODGE, 2003; PITZ FLORIANI et al., 2011).

Desta forma, a seleção de progênies ou genótipos superiores em populações de Eucalyptus é imprescindível para o estabelecimento de programas de melhoramento genético em regiões com ocorrência de neve ou geadas severas (PIRES et al., 2011). Contudo, pesquisas a respeito de tolerância ao frio em espécies arbóreas tropicais, como o E. urophylla, são escassas, além de não se ter conhecimento da variabilidade genética da espécie quanto a este evento. Desta forma, a hipótese deste trabalho sustenta-se na existência de variabilidade genética para tolerância ao frio em populações de E. urophylla, pois apesar da espécie se distribuir em latitude tropical, ocorre em grandes altitudes no seu centro de origem (MOURA, 2004; LI, et al., 2012).

Assim, o objetivo deste trabalho foi estimar a variabilidade genética para caracteres silviculturais em progênies de polinização aberta de E. urophylla para tolerância à geada em ambientes contrastantes para este evento e selecionar genótipos tolerantes ao frio.

\section{MATERIAL E MÉTODOS}

As progênies de polinização aberta de Eucalyptus urophylla utilizadas na pesquisa representam boa parte da base genética da espécie no Brasil, e foram compostas por material do Instituto de Pesquisas e Estudos Florestais (IPEF/ESALQ/USP) a partir de seis procedências: Teixeira de Freitas/ BA, Lençóis Paulista/SP, Acesita/MG, Itamarandiba/MG, Timor e Flores originárias da Indonésia, das ilhas de Timor e Flores. Historicamente, essas procedências introduzidas no país já tiveram oportunidades de se recombinar por vários momentos, em algumas décadas, possibilitando dessa forma, que se possa considerá-las como um único conjunto gênico populacional viabilizando as estimativas dos parâmetros genéticos (MISSIO et al., 2005).

Os experimentos foram instalados em 2009 em três condições ambientais (Tabela 1): nos municípios de Itatinga/SP (Empresa Eucatex S.A.) e de Palma Sola/SC (Empresa Palmasola), ambos com ocorrência de geadas e em Anhembi/SP (Estação Experimental de Anhembi, da ESALQ/USP), usado como testemunha. O clima dos locais (Anhembi/SP e Itatinga/SP), segundo a classificação de Köppen, é do tipo Cwa (temperado úmido com inverno seco e verão quente) e Cfa (Palma Sola/ SC) clima mesotérmico úmido, com verões quentes e invernos rigorosos.

Os solos foram classificados como Latossolo Bruno Distrófico, textura argilosa (Palma Sola/SC) e Neossolo Quartzarênico (Anhembi e Itatinga, SP) pela classificação da EMBRAPA (1999). O delineamento estatístico utilizado nas implantações dos testes de progênies de polinização aberta de E. urophylla foi o de blocos completos casualizados, compostos de 150 progênies, quatro repetições, seis plantas por parcela, totalizando 3.600 plantas por experimento. $\mathrm{O}$ espaçamento utilizado nos testes foi de 3 × 2 metros e bordadura externa dupla. Aos 12 e 24 meses de idade, foram avaliados em todos os testes a altura total da árvore (ALT em m), o diâmetro à altura do peito (DAP em $\mathrm{cm}$ ) e volume de madeira (VOL em $\mathrm{m}^{3}$ ). A avaliação da resistência à geada (frio) foi feita indiretamente 
avaliando-se a produtividade das plantas. Ou seja, plantas muito afetadas pelo frio produziram menos que as menos afetadas.

Tabela 1. Caracterização dos locais de implantação dos experimentos.

Table 1. Characteristics of the study sites.

\begin{tabular}{lcccccccc}
\hline Local & $\mathbf{N}^{\circ}$ de progênies & Latitude (S) & Longitude $(\mathbf{W})$ & Altitude $(\mathbf{m})$ & TMA $\left.^{\circ} \mathbf{C}\right)$ & $\mathbf{T m}\left({ }^{\circ} \mathbf{C}\right)$ & TM $\left.^{\circ}{ }^{\circ} \mathbf{C}\right)$ & PP $(\mathbf{m m})$ \\
\hline Anhembi/SP & 150 & $22^{\circ} 28^{\prime}$ & $48^{\circ} 07^{\prime}$ & 472 & 22,3 & 18,5 & 25,2 & 1300 \\
Itatinga/SP & 150 & $23^{\circ} 20^{\prime}$ & $48^{\circ} 36^{\prime}$ & 845 & 19,4 & 16,0 & 22,7 & 1500 \\
Palma Sola/SC & 150 & $26^{\circ} 20^{\prime}$ & $53^{\circ} 16^{\prime}$ & 870 & 17,4 & 15,0 & 24,4 & 2200 \\
\hline
\end{tabular}

TMA = temperatura média anual; Tm = temperatura média mínima anual; TM = temperatura média máxima anual; PP $=$ precipitação pluviométrica.

As estimativas dos componentes de variâncias e dos parâmetros genéticos foram obtidas a partir do programa estatístico SELEGEN utilizando o procedimento REML/BLUP (Estimativa dos parâmetros genéticos por máxima verossimilhança restrita - REML, e predição dos valores genéticos pelo procedimento da melhor predição linear não viciada - BLUP), conforme proposto por Resende (2007). Os parâmetros foram estimados utilizando o modelo 93 para as análises individuais de cada experimento, e para cada idade: $\mathrm{y}=\mathrm{X} r+\mathrm{Z} a+\mathrm{W} p+\varepsilon$, onde: $y$ é o vetor de dados, $r$ é o vetor dos efeitos de repetição (assumidos como fixos) somados à média geral, $a$ é o vetor dos efeitos genéticos aditivos individuais (assumidos como aleatórios), p é o vetor dos efeitos de parcela (assumidos como aleatórios) e $\varepsilon$ é o vetor de erros ou resíduos (aleatórios). As letras maiúsculas representam as matrizes de incidência para os referidos efeitos. $X, Z$ e $W$ são matrizes de incidência conhecidas, formadas por valores zero e um, as quais associam-se as incógnitas $r$, a e $p$ ao vetor de dados $y$, respectivamente. A metodologia de modelos mistos permite estimar $r$ pelo procedimento de quadrados mínimos generalizados e predizer $a$ e $p$ pelo procedimento BLUP (Best Linear Unbiased Prediction). Por meio dos algoritmos EM realiza-se o procedimento REML (Restrict Maximum Likelihood) ou método da máxima verossimilhança, onde as resoluções de matrizes geram estimativas de efeitos ajustados dos vetores calculados. Foram calculados os seguintes parâmetros genéticos:

a) Variância genética aditiva $\left(\hat{\sigma}_{a}^{2}\right)$;

$$
\hat{\sigma}_{a}^{2}=\left[\hat{a}^{\prime} A^{-1} \hat{a}+\hat{\sigma}_{\varepsilon}^{2} \operatorname{tr}\left(A^{-1} C^{22}\right)\right] / q
$$

b) Variância ambiental entre parcelas $\left(\hat{\sigma}_{c}^{2}\right)$;

$$
<\hat{\sigma}_{c}^{2}=\left[\hat{c}^{\prime} \hat{c}+\hat{\sigma}_{\varepsilon}^{2} \operatorname{tr} C^{33}\right] / s_{1}
$$

c) Variância residual (ambiental + não aditiva) $\left(\hat{\sigma}_{\varepsilon}^{2}\right)$;

$$
\hat{\sigma}_{\varepsilon}^{2}=\left[y^{\prime} y-\hat{r}^{\prime} X^{\prime} y-\hat{a}^{\prime} Z^{\prime} y-\hat{c}^{\prime} W^{\prime} y\right] /[N-r(x)]
$$

em que: $C^{22}, C^{33}, C^{44}$ e $C^{55}$ vem da inversa de $C$.

$C$ : matriz dos coeficientes das equações de modelo misto.

tr: operador traço matricial.

$r(x)$ : posto da matriz X.

$N, q, s_{1}$ : números de dados, de indivíduos e de parcelas, respectivamente.

d) Variância fenotípica individual $\left(\hat{\sigma}_{f}^{2}\right)$ :

$$
\hat{\sigma}_{f}^{2}=\hat{\sigma}_{a}^{2}+\hat{\sigma}_{c}^{2}+\hat{\sigma}_{\varepsilon}^{2}
$$

e) Herdabilidade individual no sentido restrito, ou seja, dos efeitos aditivos:

f) Herdabilidade da média de progênies:

$$
\hat{h}_{a}^{2}=\frac{\hat{\sigma}_{a}^{2}}{\hat{\sigma}_{f}^{2}} ;
$$

g) Herdabilidade aditiva dentro de parcela:

$$
\hat{h}_{m}^{2}=\frac{(1 / 4) \cdot \hat{\sigma}_{a}^{2}}{(1 / 4) \cdot \hat{\sigma}_{a}^{2}+\frac{\hat{\sigma}_{c}^{2}}{r}+\frac{\left(0,75 \cdot \hat{\sigma}_{a}^{2}+\hat{\sigma}_{\varepsilon}^{2}\right)}{n \cdot r}} ;
$$

$$
\hat{h}_{a d}^{2}=\frac{0,75 \cdot \hat{\sigma}_{a}^{2}}{0,75 \cdot \hat{\sigma}_{a}^{2}+\hat{\sigma}_{\varepsilon}^{2}} ;
$$

Sci. For., Piracicaba, v. 44, n. 110, p. 373-381, jun. 2016 DOI: dx.doi.org/10.18671/scifor.v44n110.10 
h) Coeficiente de variação genética aditiva individual:

$$
C V_{g i}(\%)=\frac{\sqrt{\hat{\sigma}_{a}^{2}}}{\hat{m}} \cdot 100 ;
$$

i) Coeficiente de variação genotípica entre progênies:

j) Coeficiente de variação experimental:

$$
C V_{g p}(\%)=\frac{\sqrt{0,25 \cdot \hat{\sigma}_{a}^{2}}}{\hat{m}} \cdot 100 ;
$$

$$
C V_{\varepsilon}(\%)=\frac{\sqrt{\left[\left(0,75 \cdot \hat{\sigma}_{a}^{2}+\hat{\sigma}_{\varepsilon}^{2}\right) / n\right]+\hat{\sigma}_{c}^{2}}}{\hat{m}} \cdot 100 ;
$$

k) Coeficiente de variação relativa:

$$
C V_{r}=\frac{C V_{g p}}{C V_{\varepsilon}}
$$

l) Acurácia da seleção de progênies, assumindo sobrevivência completa:

$$
\hat{r}_{a a}=\sqrt{\hat{h}_{m}^{2}}
$$

m) Coeficiente de determinação dos efeitos de parcela $\left(\hat{C}_{p}^{2}\right)$ :

$$
\hat{C}_{p}^{2}=\frac{\hat{\sigma}_{c}^{2}}{\hat{\sigma}_{f}^{2}}
$$

A análise conjunta foi realizada pelo modelo 04 , no delineamento experimental em blocos ao acaso, progênies de meios-irmãos, várias plantas por parcela, vários locais, para cada uma das idades avaliadas.

\section{RESULTADOS E DISCUSSÃO}

As progênies tiveram crescimento adequado aos obtidos por outros autores ao longo dos dois anos em Anhembi/SP e Itatinga/SP (Pupin et al., 2015). Similarmente à Itatinga/SP, Palma Sola/ SC, o crescimento das progênies foi inferior nas duas idades de avaliação (Tabela 2). Isto pode ter ocorrido por essas áreas apresentarem geadas mais severas. Pois, mesmo com progênies tolerantes a esta condição, a produtividade é menor.

Tabela 2. Estimativas dos parâmetros genéticos para as características de crescimento em altura de planta (ALT, $\mathrm{m})$, diâmetro à altura do peito (DAP, $\mathrm{cm})$, volume de madeira $\left(\mathrm{VOL}, \mathrm{m}^{3}\right)$ nas idades de 12 e 24 meses para os testes de progênies de $E$. urophylla para a localidade de Anhembi/SP (sem ocorrência de danos

\begin{tabular}{|c|c|c|c|c|c|c|c|c|c|c|c|c|c|}
\hline \multicolumn{14}{|c|}{ Parâmetros genéticos } \\
\hline \multirow{2}{*}{$\begin{array}{l}\text { Caracte- } \\
\text { rísticas }\end{array}$} & \multirow{2}{*}{ Local } & \multicolumn{2}{|c|}{ Média } & \multicolumn{2}{|c|}{ LTR } & \multicolumn{2}{|c|}{$\hat{C}_{\text {parc }}^{2}$} & \multicolumn{2}{|c|}{$C V_{\varepsilon \%}$} & \multicolumn{2}{|c|}{$\mathrm{CV}_{\mathrm{gi}} \%$} & \multicolumn{2}{|c|}{ CVgp\% } \\
\hline & & $12^{*}$ & 24 & 12 & 24 & 12 & 24 & 12 & 24 & 12 & 24 & 12 & 24 \\
\hline & Anhembi & 10,40 & 14,99 & $165,89^{* *}$ & $177,21^{* *}$ & 0,123 & 0,065 & 11,52 & 10,65 & 10,81 & 11,71 & 5,40 & 5,85 \\
\hline \multirow[t]{3}{*}{ ALT } & Itatinga & 6,79 & 10,68 & $437,25^{\star *}$ & $300,06^{* *}$ & 0,126 & 0,031 & 11,02 & 9,35 & 11,67 & 16,61 & 5,83 & 8,30 \\
\hline & Palma Sola & 3,79 & & $135,03^{* *}$ & & 0,123 & 0,132 & 23,11 & 23,51 & 16,22 & 16,26 & 8,13 & 8,13 \\
\hline & Anhembi & 7,89 & 10,52 & $110,51^{* *}$ & $162,02^{* *}$ & 0,045 & 0,006 & 12,61 & 12,34 & 11,53 & 13,04 & 5,76 & 6,52 \\
\hline \multirow[t]{3}{*}{ DAP } & Itatinga & 5,65 & 8,80 & $432,35^{* *}$ & $251,24^{* *}$ & 0,018 & 0,005 & 9,80 & 10,98 & 17,90 & 21,14 & 8,99 & 10,56 \\
\hline & Palma Sola & 7,09 & 10,60 & $146,42^{* *}$ & $171,07^{* *}$ & 0,102 & 0,101 & 9,41 & 9,50 & 6,98 & 7,00 & 3,49 & 3,51 \\
\hline & Anhembi & 0,029 & 0,077 & $109,42^{* *}$ & $129,66^{* *}$ & 0,061 & 0,008 & 27,26 & 25,94 & 27,51 & 28,06 & 13,75 & 14,03 \\
\hline \multirow[t]{2}{*}{ VOL } & Itatinga & 0,010 & 0,047 & $358,08^{* *}$ & $226,84^{* *}$ & 0,073 & 0,034 & 23,63 & 21,51 & 43,39 & 45,16 & 21,69 & 22,58 \\
\hline & Palma Sola & 0,008 & 0,012 & $80,64^{* *}$ & $102,83^{* *}$ & 0,112 & 0,114 & 36,04 & 36,10 & 31,35 & 31,40 & 15,67 & 15,70 \\
\hline
\end{tabular}
por geada), e para as localidades de Itatinga/SP e Palma Sola/SC (com ocorrência de danos por geada).

Table 2. Estimates of genetic parameters for growth characteristics in plant height (ALT $m$ ), diameter at breast

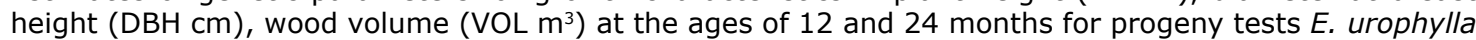
to the location of Anhembi / SP (no occurrence of frost damage), and the locality of Itatinga / SP and Palma Sola / SC (with occurrence of frost damage).

Média * 12 e 24 meses de idade; LTR= teste da razão de verossimilhança (***ignificativo a I\%, com 0,5 grau de liberdade; ns não significativo); $C_{p a r c}^{2}$ : coeficiente de determinação dos efeitos de parcela; $C V_{e} \%$ : coeficiente de variação residual; $C V$ gi $\%:$ coeficiente de variação genética aditiva individual; $C V_{g p} \%$ : coeficiente de variação genotípica entre progênies.

Em relação a Anhembi, onde não houve geada, em Itatinga, com a ocorrência da geada em nível moderado (temperatura variando entre $+2 \mathrm{a}+3^{\circ} \mathrm{C}$ (Informação pessoal do autor), o crescimento das progênies foi menos afetado, se comparado com o observado em Palma Sola onde as geadas 
atingiram temperaturas próximas a $-10^{\circ} \mathrm{C}$ (MORAES et al., 2014) para o ano de avaliação (geadas extremas). Importante enfatizar que, em povoamentos próximos à área experimental em Palma Sola, observou-se que até as espécies subtropicais como E. benthamii e E. dunnii, em condições de geadas extremas como ocorridas em 2008 e 2010, sofreram danos, mas em percentagens menores quando comparadas com espécies tropicais como E. urophylla, E. grandis, E. saligna e outras (Informações pessoais).

Diferentes híbridos de Eucalyptus (E. saligna; E. torelliana; híbridos de E. urophylla x E. grandis, E. grandis $\mathrm{x}$ E. camaldulensis e híbridos espontâneos de E. dunnii) também em Palma Sola/SC apresentaram crescimento médio, de 10,35 m e 11,62 cm para altura de plantas e DAP aos 30 meses de idade, mesmo após os danos sofridos pela geada (MORAES et al., 2011). Tais resultados confirmam que a produção de madeira de eucaliptos com qualidade para essas condições climáticas adversas é possível, mas a escolha de espécies e/ou genótipos mais resistentes é necessária.

O coeficiente de variação experimental $\left(\mathrm{CV}_{\varepsilon} \%\right)$ para VOL foi superior aos observados para DAP e ALT, mas ainda está adequado para experimentos de campo com E. urophylla (Tabela 2). As estimativas do ${ }^{C V_{\varepsilon}}$ variaram pouco nas duas idades, para cada característica, e foram semelhantes entre DAP e ALT, exceto para ALT em Palma Sola, cujos valores foram superiores. De acordo com Garcia (1989), os coeficientes de variação experimental para espécies florestais, em condições de campo, mesmo em valores superiores aos observados para outras culturas, espécies e gêneros, revelam boa precisão experimental.

Os valores dos coeficientes de variação experimental ( $\left.\mathrm{CV}_{\varepsilon} \%\right)$ permaneceram entre 9,35\% a 36,10\%, o que são adequados para testes de progênies de espécies florestais. Mori et al. (1988), Paula et al. (2002), Rocha et al. (2007), Freitas et al. (2009) e Pinto et al. (2014), estudando as espécies E. grandis, E. camaldulensis, E. urophylla e E. dunni em regiões sem ocorrências de geadas encontraram resultados similares para o $\mathrm{CV}_{\varepsilon} \%$. Em E. dunnii atingido por geadas, em Campo do Tenente/PR, as estimativas de coeficientes de variação experimental $\left(\mathrm{CV}_{\varepsilon} \%\right)$ para altura de plantas, DAP e volume de madeira, aos 12, 24 e 36 meses, variaram de 13,15\% a 41,26\% (HIGA, 1998), portanto, superiores às obtidas no presente trabalho. As estimativas dos coeficientes de variação genética $\left(\mathrm{CV}_{g} \%\right)$, tanto a nível individual $\left(\mathrm{CV}_{g i} \%\right)$ quanto de médias de progênies $\left(\mathrm{CV}_{g g} \%\right)$ para VOL, 27,51\% a $45,16 \%$, indicam ótimo potencial para a seleção individual ou de média de famílias, mesmo para condições de geadas (Tabela 2). Segundo Sebbenn et al. $(1998,1999)$ o coeficiente de variação genética é um parâmetro importante para os programas de melhoramento vegetal, por permitir um ganho adicional pela seleção dentro de progênies. Os valores das estimativas do coeficiente de variação genética dentro de progênies reforçam a hipótese da eficiência de ganhos em trabalhos de genética florestal (SOUZA et al., 2011).

Para as três características analisadas, as estimativas do coeficiente de determinação dos efeitos ambientais entre parcelas ( $\hat{C}_{p a r c}^{2}$, Tabela 2 ), foram de magnitude baixa a moderada, ou seja, o ambiente atuou pouco entre as parcelas, promovendo baixa variação ambiental entre as parcelas em todos os locais. Rocha et al. (2006), estudando o gênero Eucalyptus, encontraram resultados semelhantes para a altura de plantas (ALT) e diâmetro à altura do peito (DAP). Segundo Resende (2002), o $\hat{C}_{p a r c}^{2}$ quantifica a variabilidade das parcelas dentro dos blocos, sendo que uma estimativa até $10 \%$ não chega a interferir na estimativa dos parâmetros genéticos.

A relação entre o coeficiente de variação genética e o coeficiente de variação experimental $\left(\mathrm{CV}_{r}\right.$, Tabela 2), estimada para ALT, DAP e VOL nos três locais e nas duas idades, indica sempre valores mais altos para o VOL em Itatinga, sugerindo que esta é a melhor condição para a seleção de indivíduos superiores em regiões com geadas fracas. Quando a relação do coeficiente de variação genético e o coeficiente de variação ambiental aproximam-se de valores maiores ou iguais a um, caracteriza-se uma situação favorável para a prática da seleção (VENCOVSKY, BARRIGA 1992).

As estimativas de herdabilidade ao nível de média de progênies $\left(\hat{h}_{m}^{2}\right)$ em Itatinga foram superiores às obtidas em Anhembi e Palma Sola (Tabela 3).

As herdabilidades no sentido restrito ao nível de plantas individuais $\left(\hat{h}_{a}^{2}\right)$, em Palma Sola $(0,13$ a 0,19 ) foram inferiores às obtidas em Anhembi e Itatinga, evidenciando o efeito do ambiente sobre as características. Comportamento semelhante foi observado para a herbabilidade dentro das parcelas $\left(\hat{h}_{a d}^{2}\right)$ e da média de progênies $\left(\hat{h}_{m}^{2}\right)$. 
Tabela 3. Estimativas dos parâmetros genéticos para as características de crescimento em altura de planta (ALT, $\mathrm{m})$, diâmetro à altura do peito (DAP, $\mathrm{cm})$, volume de madeira $\left(\mathrm{VOL}, \mathrm{m}^{3}\right)$ nas idades de 12 e 24 meses para os testes de progênies de $E$. urophylla para a localidade de Anhembi/SP (sem ocorrência de danos por geada), e para as localidades de Itatinga/SP e Palma Sola/SC (com ocorrência de danos por geada).

Table 3. Estimates of genetic parameters for growth characteristics in plant height (ALT $\mathrm{m}$ ), diameter at breast height $(\mathrm{DBH} \mathrm{cm})$, wood volume $\left(\mathrm{VOL} \mathrm{m}{ }^{3}\right)$ at the ages of 12 and 24 months for progeny tests of $E$. urophylla in Anhembi / SP (no occurrence of frost damage), and the locality of Itatinga / SP and Palma Sola / SC (with occurrence of frost damage).

\begin{tabular}{|c|c|c|c|c|c|c|c|c|c|c|c|}
\hline \multicolumn{12}{|c|}{ Parâmetros genéticos } \\
\hline \multirow{2}{*}{ Características } & \multirow{2}{*}{ Local } & \multicolumn{2}{|c|}{$\hat{h}_{a}^{2}$} & \multicolumn{2}{|c|}{$\hat{h}_{m}^{2}$} & \multicolumn{2}{|c|}{$\hat{h}_{a d}^{2}$} & \multicolumn{2}{|c|}{$C V_{r}$} & \multicolumn{2}{|c|}{$\hat{r}_{a a}(\%)$} \\
\hline & & $12^{*}$ & 24 & 12 & 24 & 12 & 24 & 12 & 24 & 12 & 24 \\
\hline & Anhembi & 0,23 & 0,25 & 0,47 & 0,55 & 0,21 & 0,22 & 0,47 & 0,55 & 68 & 74 \\
\hline \multirow[t]{3}{*}{ ALT } & Itatinga & 0,63 & 0,54 & 0,72 & 0,76 & 0,66 & 0,48 & 0,80 & 0,88 & 84 & 87 \\
\hline & Palma Sola & 0,13 & 0,13 & 0,32 & 0,31 & 0,12 & 0,11 & 0,35 & 0,37 & 57 & 58 \\
\hline & Anhembi & 0,16 & 0,18 & 0,45 & 0,53 & 0,13 & 0,14 & 0,45 & 0,53 & 67 & 73 \\
\hline \multirow[t]{3}{*}{ DAP } & Itatinga & 0,53 & 0,55 & 0,77 & 0,79 & 0,47 & 0,48 & 0,91 & 0,96 & 88 & 88 \\
\hline & Palma Sola & 0,13 & 0,13 & 0,35 & 0,35 & 0,11 & 0,12 & 0,38 & 0,36 & 59 & 60 \\
\hline & Anhembi & 0,21 & 0,19 & 0,50 & 0,54 & 0,18 & 0,15 & 0,50 & 0,54 & 71 & 73 \\
\hline \multirow[t]{2}{*}{ VOL } & Itatinga & 0,67 & 0,72 & 0,77 & 0,81 & 0,66 & 0,69 & 0,91 & 1,04 & 92 & 90 \\
\hline & Palma Sola & 0,19 & 0,19 & 0,43 & 0,43 & 0,17 & 0,17 & 0,44 & 0,43 & 66 & 67 \\
\hline
\end{tabular}

$\hat{h}_{a}^{2}:$ herdabilidade individual no sentido restrito; $\hat{h}_{m}^{2}:$ herdabilidade da média de progênies; $\hat{h}_{a d}^{2}:$ herdabilidade aditiva dentro de parcela; $C V_{r}$ : coeficiente de variação relativa; $\hat{r}_{a s}$ acurácia da seleção de progênies; $12 *$ e 24 meses de idade.

Os valores de $\hat{h}_{m}^{2}$, para Itatinga, superiores a 0,72 , independentemente da característica, nas duas idades, revelam alto controle genético e boas perspectivas para a seleção de famílias. Estes valores apresentam-se altos, pois o material genético de E. urophylla encontra-se em fase inicial do programa de melhoramento. Os valores de herdabilidade ao nível de média de progênies para DAP e ALT estão de acordo com os resultados obtidos por Kageyama e Vencovsky (1983), em progênies de E. grandis aos 30 anos de idade. Martins et al. (2001), em estudo com progênies de meios irmãos de E. grandis, encontraram valores de herdabilidade média de progênies para ALT e DAP iguais a 0,68 e 0,69 respectivamente, sendo valores menores que os encontrados neste estudo. Zanata et al. (2010), estudando Eucalyptus pellita, encontraram valores de $\hat{h}_{m}^{2}$ de 0,85 para ALT e 0,90 para DAP, valores esses superiores aos obtidos no presente trabalho.

A acurácia seletiva pode ser considerada adequada para todas as características estudadas, chegando a 92\% (VOL). Isso indica boa precisão no acesso à variação genética verdadeira a partir da variação fenotípica observada para as características (Tabela 3). O valor da acurácia seletiva superior a 50\% ( $\left.\hat{r}_{a a}\right)$ é ideal para a seleção conforme relatado por Resende e Duarte (2007), indicando maior eficiência na seleção dos genótipos de interesse.

As análises de deviance para todas as variáveis, indicam a existência de diferença entre progênies e a possibilidade de ganhos com a seleção com e sem a presença de geadas. Os efeitos da interação genótipos $\mathrm{x}$ ambientes foram significativos, indicando diferenças de desempenho dos genótipos nos ambientes, como por exemplo em condições de geada.

Para a escolha das progênies que apresentam adaptação ampla ou restrita aos locais específicos (BRESSIANI et al., 2002), estudos mais detalhados dessa interação são necessários, pois esses valores servirão de subsídios aos melhoristas. O coeficiente de determinação dos efeitos de parcelas $\left(\hat{C}_{p a r c}^{2}\right)$ foi baixo para todas as características, indicando que não houve heterogeneidade ambiental dentro dos blocos, podendo-se inferir que o delineamento foi eficiente e a capacidade de teste adequada (STURION; RESENDE, 2005). A acurácia seletiva para ALT, DAP e VOL foi acima de 76\%, indicando boa perspectiva de seleção e de ganhos genéticos.

O resultado do $\hat{C}_{\text {int }}^{2}$ conduziu a um valor de correlação genotípica $\left(\hat{r}_{g l o c}\right)$ acima de $60 \%$ para ALTe DAP para ambas as idades, apresentando boa magnitude, indicando que a interação não é de natureza complexa, ou seja, a posição relativa dos genótipos é pouco alterada entre os ambientes (VENCOVSKY; BARRIGA, 1992; ZIMBACK et al., 2011). A interação simples ocorre quando os genótipos apresentam diferentes comportamentos nos diferentes tipos de ambiente, mas a classificação da produtividade dos genótipos não altera entre os ambientes (MORAIS et al., 2010), sendo provocada pela diferença de variabilidade genética entre as progênies. 
Tabela 4. Análise de deviance para as características altura de plantas (ALT, $m$ ), diâmetro à altura do peito (DAP, $\mathrm{cm}$ ) e volume de madeira (VOL, $\mathrm{m}^{3}$ ) em progênies de $E$. urophylla, em três locais (Anhembi/SP, Itatinga/ SP e Palma Sola/SC), aos $12 *$ e 24 meses de idade.

Table 4. Deviance analysis for the quantitative characteristics height (ALT, $m$ ) diameter at breast height (DAP, $\mathrm{cm}$ ) and wood volume $\left(\mathrm{VOL}, \mathrm{m}^{3}\right.$ ) in E. urophylla progenies, for the three locations (Anhembi/SP, Itatinga/SP e Palma Sola/SC), at $12 *$ and 24 months of age.

\begin{tabular}{lcccccc}
\hline \multirow{2}{*}{ Efeito } & \multicolumn{2}{c}{ ALT } & \multicolumn{2}{c}{ DAP } & \multicolumn{2}{c}{ VOL } \\
\cline { 2 - 7 } & $\mathbf{1 2}^{*}$ & $\mathbf{2 4}$ & $\mathbf{1 2}$ & $\mathbf{2 4}$ & $\mathbf{1 2}$ & $\mathbf{2 4}$ \\
\hline Progênies & $359,16^{* *}$ & $320,32^{* *}$ & $371,57^{* *}$ & $389,83^{* *}$ & $270,40^{* *}$ & $234,84^{* *}$ \\
Parcela & $105,66^{* *}$ & $5,71^{*}$ & $9,78^{* *}$ & $0,45 \mathrm{~ns}$ & $0,50 \mathrm{~ns}$ & $0,36 \mathrm{~ns}$ \\
Prog x Loc & $98,08^{* *}$ & $85,71^{* *}$ & $53,34^{* *}$ & $14,68^{* *}$ & $15,30^{* *}$ & $91,58^{* *}$ \\
\hline
\end{tabular}

Qui-quadrado tabelado: $3,84\left(^{*}\right)$ e $6,63\left(^{* *}\right)$ para os níveis de significância de $5 \%$ e $1 \%$, respectivamente, a I grau de liberdade.

Tabela 5. Estimativas dos parâmetros genéticos na análise conjunta dos três locais dos testes de progênies de $E$. urophylla, avaliados aos 12 e 24 meses de idade, para as características altura de plantas (ALT m), diâmetro à altura do peito (DAP $\mathrm{cm})$ e volume de madeira $\left(\mathrm{VOL} \mathrm{m}^{3}\right)$.

Table 5. Estimates of genetic parameters for the joint analysis of the three edaphic-climatic regions where $E$. urophylla progeny tests were assessed at 12 and 24 months of age; for the characteristics: plant height (ALT $\mathrm{m}$ ) diameter at breast height (DAP $\mathrm{cm})$ and wood volume $\left(\mathrm{VOL} \mathrm{m}^{3}\right)$.

\begin{tabular}{|c|c|c|c|c|c|c|}
\hline \multirow{3}{*}{ Parâmetros genéticos } & \multicolumn{6}{|c|}{ Características } \\
\hline & \multicolumn{2}{|c|}{ ALT } & \multicolumn{2}{|c|}{ DAP } & \multicolumn{2}{|c|}{ VOL } \\
\hline & $12^{*}$ & 24 & 12 & 24 & 12 & 24 \\
\hline$\widehat{\hat{h}_{a}^{2}}$ & 0,056 & 0,060 & 0,043 & 0,055 & 0,035 & 0,048 \\
\hline$\hat{C}_{p a r t}^{2}$ & 0,100 & 0,070 & 0,079 & 0,012 & 0,066 & 0,014 \\
\hline$\hat{C}_{\mathrm{in}}^{2}$ & 0,016 & 0,017 & 0,028 & 0,022 & 0,025 & 0,028 \\
\hline$\hat{h}_{m}^{2}$ & 0,68 & 0,72 & 0,60 & 0,72 & 0,60 & 0,70 \\
\hline$\hat{r}_{a a}$ & 0,82 & 0,85 & 0,78 & 0,85 & 0,76 & 0,82 \\
\hline$\hat{r}_{g l o c}$ & 0,77 & 0,78 & 0,60 & 0,71 & 0,58 & 0,63 \\
\hline$C V_{g p}(\%)$ & 6,5 & 6,71 & 5,05 & 6,22 & 13,42 & 15,82 \\
\hline $\mathrm{CV}_{\varepsilon}$ & 13,68 & 12,58 & 11,38 & 10,75 & 32,71 & 29,32 \\
\hline Média & 6,61 & 10,46 & 7,26 & 9,99 & 0,0158 & 0,0458 \\
\hline
\end{tabular}

Qui-quadrado tabelado: $3,84\left({ }^{*}\right)$ e $6,63\left(^{* *}\right)$ para os níveis de significância de $5 \%$ e I\%, respectivamente, a I grau de liberdade.

As estimativas dos coeficientes de herdabilidade em nível de média de progênies $\left(\hat{h}_{m}^{2}\right)$ foram altas, acima de $60 \%$ para as três características, indicando que grande parte da variação fenotípica total e média entre progênies é de origem genética e, portanto, o controle genético das características é alto ao nível de média de progênies na análise conjunta.

\section{CONCLUSÕES}

O material genético de E. urophylla avaliado na presente pesquisa possui variabilidade genética para tolerância à geada, mostrando potencialidade para seleção em programas de melhoramento. A interação genótipos $\mathrm{x}$ ambientes $(\mathrm{GxE})$ é de natureza, predominantemente, simples, indicando diferenças de variabilidade entre as progênies nos três ambientes.

\section{AGRADECIMENTOS}

À Coordenação de Aperfeiçoamento de Pessoal de Nível Superior (CAPES), pela concessão de bolsa de estudos ao primeiro autor, às empresas Eucatex Florestal S/A, Palmasola S/A e à Estação Experimental de Ciências Florestais de Anhembi pelo fornecimento das áreas de estudos e ao Instituto de Pesquisas e Estudos Florestais (IPEF) pelo fornecimento das progênies estudadas.

\section{REFERÊNCIAS BIBLIOGRÁFICAS}

ALMEIDA, A. C.; SOARES, J. V.; LANDSBERG J. J.; REZENDE, G. D. Growth and water balance of Eucalyptus grandis hybrid plantations in Brazil during a rotation for pulp production. Forest Ecology and Management, Amsterdam, v. 251, n. 1-2, p. 10-21, 2007. 
Moraes et al. - Controle genético para a tolerância a geada em progênies de Eucalyptus urophylla

BRESSIANI, A. J.; VENCOVSKY, R.; BURNQUIST, W. L. Interação entre famílias de cana-de-açúcar e locais: efeito na resposta esperada com a seleção. Bragantia, Campinas, v. 61, n. 1, p. 1-10, 2002.

EMBRAPA. EMPRESA BRASILEIRA DE PESQUISA AGROPECUÁRIA. Sistema brasileiro de classificação de solos. Brasília, 1999. 412 p.

FREITAS, R. G.; VASCONCELOS, E. S.; CRUZ, C. D.; ROSADO, A. M.; ROCHA, R. B.; TAKAMI, L. K. Predição de ganhos genéticos em progênies de polinização aberta de Eucalyptus urograndis cultivadas em diferentes ambientes e submetidas a diferentes procedimentos de seleção. Revista Árvore, Viçosa, v. 33, n. 2, p. 255-263, 2009.

GARCIA, C. H. Tabelas para classificação do coeficiente de variação. Piracicaba: IPEF, 1989, 10 p. (Circular Técnica, 171).

HIGA, R. C. V. Avaliação e recuperação de Eucalyptus dunnii Maiden atingidos por geadas em Campo do Tenente, PR. 1998. 100 p. Tese (Doutorado em Silvicultura) - Universidade Federal do Paraná, Curitiba, 1998.

KAGEYAMA, P. Y.; VENCOVSKY, R. Variação genética em progênies de uma população de Eucalyptus grandis (Hill) Maiden. IPEF, Piracicaba, n. 24, p. 9-26, 1983.

KAYAL, W. E.; NAVARRO, M.; MARQUE, G.; KELLER, G.; MARQUE, C.; TEULIERES, C. Expression profile of CBF-like transcriptional factor genes from Eucalyptus in response to cold. Journal of Experimental Botany, Oxford, v. 57, n. 10, p. 2455-2469, 2006.

MARTINS, I. S.; MARTINS, R. C. C.; CORREIA, H. S. Comparação entre seleção combinada e seleção direta em Eucalyptus grandis, sob diferentes intensidades de seleção. Floresta e Ambiente, Seropédica, v. 8, n. 1, p. 36-43, 2001.

MISSIO, R. F.; SILVA, A. M.; DIAS, L. A. S.; MORAES, M. L. T.; RESENDE, M. D. V. Estimates of genetic parameters and prediction of additive genetic values in Pinus kesya progenies. Crop Breeding and Applied Biotechnology, Viçosa, v. 5, n. 4, p. 394-401, 2005.

MORAES, C. B.; FREITAS, T. C. M.; PIERONI, G. B.; RESENDE, M. D. V.; ZIMBACK, L.; MORI, E. S. Estimativas de parâmetros genéticos para seleção precoce de clones de Eucalyptus para região com ocorrência de geadas. Scientia Forestalis, Piracicaba, v. 42, n. 102, p. 219-227, 2014.

MORAES, C. B.; FREITAS, T. C. M.; PIERONI, G. B.; ZIMBACK, L.; MORI, E. S. Genetic variability in eucalypt for frost tolerance. In: IUFRO WORKING GROUP IMPROVEMENT AND OF CULTURE EUCALYPTUS, Porto Seguro: IUFRO, 2011.

MORI, E. S.; KAGEYAMA, P. Y.; FERREIRA, M. Variação genética e interações progênies x locais em Eucalyptus urophylla. IPEF, Piracicaba, n. 39, p. 53-63, 1988.

MOURA, V. P. G. O germoplasma de Eucalyptus urophylla S. T. Blake no Brasil. Brasília: Embrapa Recursos Genéticos e Biotecnologia, 2004. 12 p. (Comunicado Técnico, 111).

PAULA, R. C.; PIRES, I. E.; BORGES, R. C. G.; CRUZ, C. D. Predição de ganhos genéticos em melhoramento florestal. Pesquisa Agropecuária Brasileira, Brasília, v. 37, n. 2, p. 159-165, 2002.

PINTO, C. S.; COSTA, R. M. L.; MORAES, C. B.; PIERI, C.; TAMBARUSSI, E. V.; FURTADO, E. L., MORI, E. S. Genetic variability in progenies of Eucalyptus dunnii Maiden for resistance to Puccinia psidii. Crop Breeding and Applied Biotechnology, Viçosa, n. 14, n. 3, p. 187-193, 2014.

PIRES, I. E.; RESENDE, M. D. V.; SILVA, R. L.; RESENDE JR., M. F. R. Genética Florestal. Viçosa: UFV, 2011. $318 \mathrm{p}$.

PITZ FLORIANI, M. M.; STEFFENS, C. A.; CHAVES, D. M. Rustificação de plantas de Eucalyptus dunnii Maiden e a relação entre as concentrações de carboidratos solúveis totais e de prolina foliar e a tolerância ao frio. Revista Árvore, Viçosa, v. 35, n. 1, p. 21-29, 2011. 
RESENDE, M. D. V. Genética biométrica e estatística no melhoramento de plantas perenes. Colombo: Embrapa Florestas, 2002. 975 p.

RESENDE, M. D. V. SELEGEN-REML/BLUP: Sistema estatístico e seleção genética computadorizada via modelos lineares mistos. Colombo: Embrapa Florestas, 2007. 359 p.

RESENDE, M. D. V.; DUARTE, J. B. Precisão e controle de qualidade em experimentos de avaliação de cultivares. Pesquisa Agropecuária Tropical, Goiânia, v. 37, n. 3, p. 182-194, 2007.

ROCHA, M. G. B.; PIRES, I. E.; ROCHA, R. B.; XAVIER, A.; CRUZ, C. D. Avaliação genética de progênies de meio-irmãos de Eucalyptus grandis por meio dos procedimentos Reml/Blup e da Anova. Scientia Forestalis, Piracicaba, v. 34, n. 71, p. 99-107, 2006.

ROCHA, M. G. B.; PIRES, I. E.; ROCHA, R. B.; XAVIER, A.; CRUZ, C. D. Seleção de genitores de Eucalyptus grandis e de Eucalyptus urophylla para produção de híbridos interespecíficos utilizando reml/ blup e informação de divergência genética. Revista Árvore, Viçosa, v. 31, n. 6, p. 977-987, 2007.

SEBBENN, A. M.; SIQUEIRA, A. C. M. F.; KAGEYAMA, P. Y.; DIO JR., O. J. Variação genética entre e dentro de populações de amendoim - Pterogyne nitens. Scientia Forestalis, Piracicaba, n. 56, p. 29-40, 1999.

SEBBENN, A. M.; SIQUEIRA, A. C. M. F.; KAGEYAMA, P. Y.; MACHADO, J. A. R. Parâmetros genéticos na conservação da cabreúva: Myroxylon peruiferum L. F. Allemão. Scientia Forestalis, Piracicaba, n. 53, p. 31-38, 1998.

SILVA, A. L. L.; OLIVEIRA, Y.; ALCANTARA, G. B.; SANTOS, M.; QUOIRIN, M. Tolerância ao resfriamento e congelamento de folhas de eucalipto. Biociências, Porto Alegre, v. 17, n. 1, p. 86-90, 2009.

SNIF. SISTEMA NACIONAL DE INFORMAÇÕES FLORESTAIS. Disponível em: <http://www.florestal.gov.br/ snif/recursos-florestais/as-florestas-plantadas/> Acesso em: 25 out. 2015.

SOUZA, C. S.; FREITAS, M. L. M.; MORAES, M. L. T.; SEBBENN, A. M. Estimativas de parâmetros genéticos para caracteres quantitativos em progênies de polinização aberta de Eucalyptus urophylla. Floresta, Curitiba, v. 41, n. 4, p. 847-856, 2011.

STURION, J. A.; RESENDE, M. D. V. Seleção de progênies de erva-mate (Ilex paraguariensis St. Hil.) para produtividade, estabilidade e adaptabilidade temporal de massa foliar. Boletim de Pesquisa Florestal, Colombo, n. 50, p. 37-51, 2005.

TAIZ, L.; ZEIGER, E. Fisiologia Vegetal. 5.ed. Porto Alegre: Artmed, 2013. 819 p.

TIBBITS, W. N.; HODGE, G. R. Genetic parameters for cold hardiness in Eucalyptus nitens (Deane \& Maiden) Maiden. Silvae Genetica, Frankfurt, v. 52, n. 3/4, p. 89-97, 2003.

VENCOVSKY, R.; BARRIGA, P. Genética biométrica no fitomelhoramento. Ribeirão Preto: Sociedade Brasileira de Genética, 1992. 416 p.

ZANATA, M.; FREITAS, M. L. M.; SILVA, M. T.; MORAIS, E.; ZANATTO, A. C. S.; SEBBENN, A. M. Parâmetros genéticos e ganhos na seleção em teste de progênies de polinização aberta de Eucalyptus pellita, em Batatais SP. Revista do Instituto Florestal, São Paulo, v. 22, n. 2, p. 233-242, 2010.

ZIMBACK, L.; MORI, E. S.; BRIZOLLA, T. F.; CHAVES, R. Correlações entre caracteres silviculturais durante o crescimento de Eucalyptus grandis Hill ex Maiden. Revista do Instituto Florestal, São Paulo, v. 23, n. 1, p. 57-67, 2011.

Recebido em 01/07/2015

Aceito para publicação em 09/11/2015

Sci. For., Piracicaba, v. 44, n. 110, p. 373-381, jun. 2016

DOI: dx.doi.org/10.18671/scifor.v44n110.10 
